\title{
Trajectory length of pitch vs. roll: Technique for assessment of postural stability
}

\author{
Patrik Kutílek$^{1}$, Vladimír Socha ${ }^{1, *}$, Ondřej Čakrtt ${ }^{1,2}$, Jakub Schlenker ${ }^{1}$, and Lucia Bizovská ${ }^{3}$ \\ ${ }^{l}$ Faculty of Biomedical Engineering, Czech Technical University, Prague, Czech Republic; ${ }^{2}$ University Hospital Motol - $2^{\text {nd }}$ \\ Faculty of Medicine, Charles University, Prague, Czech Republic; and ${ }^{3}$ Faculty of Physical Culture, Palacký University \\ Olomouc, Olomouc, Czech Republic
}

Copyright: (c) 2015 P. Kutílek et al. This is an open access article licensed under the Creative Commons Attribution License (http://creativecommons.org/licenses/by/4.0/).

\begin{abstract}
Background: Patients with disorders of nervous or musculoskeletal system often show instability of the body segments during the stance tasks. Traditionally, stabilometric platforms are used to measure body sway. However, these devices are expensive and do not allow the evaluation of the individual movements of body segments. At present, accelerometers or gyroscopes are used to measure the movements of the body segments. For these new motion capture (MoCap) systems, methods for quantitative evaluation of a body segment movement are being developed. Objective: The main objective of this paper is to describe a new method which would be suitable for quantifying postural stability and identifying differences in balance control using data recorded by an inexpensive 2-DoF gyroscope. Methods: Method based on total length of trajectory (TL) in a 2-D plot of angles was proposed for quantitative evaluation of the trunk and feet sway. The sway was measured during quiet stance of ten middle-aged patients (Pts) with degenerative cerebellar disorder and eleven young healthy subjects (HS) standing with eyes open (EO) on a firm surface (FiS) and eyes closed (EC) on a foam surface (FoS). Data were obtained using three gyroscopes (Xsens) to measure roll and pitch angular movements of the trunk, and left and right foot. The pitch versus roll plots of the trunk and feet were created and the trajectory lengths of the pitch vs. roll angle were calculated. Results: Although the results vary while measuring different segments of the body, the method showed significant differences between the two different groups. Significant differences between the HS and Pts were found in EO standing on a FiS for TLs of the trunk $(p=.02)$ and TLs of the feet $(p<.01)$. Similarly in EC standing on a FoS significant differences $(p<.01)$ between groups were found for TLs of both the trunk and the feet $(p<.01)$. Conclusions: It was found that the TL of pitch vs. roll is suitable for quantifying postural sway and identifying differences in balance control. The technique, based on the length of the pitch angle vs. roll angle trajectory can be used even where MoCap systems can only measure two sway angles which could extend the clinical information of the body sway.
\end{abstract}

Keywords: trunk sway, feet sway, postural stability, length of trajectory, pitch vs. roll, cerebellar disorder

\section{Introduction}

Many of disorders, such as disorders of nervous or musculoskeletal system (Duclos, Nadeau, \& Lecours, 2008), can negatively affect the position and orientation of the body segments. Patients with these disorders often show instability of the body segments during stance tasks. Making the stance task more difficult by removing visual inputs has been claimed as a means to identify specific deficits (Morris, 2000).

\footnotetext{
* Address for correspondence: Vladimír Socha, Faculty of Biomedical Engineering, Czech Technical University, nám. Sítná 3105, 27201 Kladno, Czech Republic. E-mail: vladimir.socha@fbmi.cvut.cz
}

Traditionally, stabilometric platforms are used to measure the body sway (Hong, Manor, \& Li, 2007; Kammermeier, Kleine, Eggert, Krafczyk, \& Büttner, 2013). However, these devices are expensive and do not allow the evaluation of the individual movements of a segment. At present, accelerometers or gyroscopes are used to measure the movements of segments. For these new motion capture systems, methods for quantitative evaluation of a movement of segment are being developed. One, two or three-axial sensors for orientation measurement (i.e. gyroscope systems) can be used for high-accuracy measurement of angular movements of the body segments. In the measurement theory, gyroscope systems measure the pitch, roll and yaw of a body segment with respect to the earth's gravity and 
horizontal reference plane. Thus the pitch, roll and yaw are not joint angles, but rather Euler angles. These angles can be used to study the orientation of body segments, whose orientation is result of a complex kinematic chain. Therefore, the pitch, roll and yaw angles are also used in medicine (Adkin, Bloem, \& Allum, 2001; Allum, Adkin, Carpenter, Held-Ziolkowska, Honegger, \& Pierchala, 2001; Gill et al., 2001). In these studies, pitch and roll sway measurements during specific balance tasks identified differences in balance control in patients (Pts), compared to those of healthy subjects (HS).

As for the data processing methods for assessment of body sway, the most commonly used methods for the assessment of postural stability are methods based on evaluation of the range of movement, mean sway velocity (Horlings et al., 2008), and frequency domain (Hwang, Huang, Cherng, \& Huang, 2006). More complex methods for assessing the postural instability are based on the shape of the envelope, confidence ellipse (Oliveira, Simpson, \& Nadal, 1996) or the trajectory length (Raymakers, Samson, \& Verhaar, 2005). These methods are based on the description of the behavior of two kinematic variables. Some of these methods were only used to evaluate data from the stabilometric platforms. Most of these methods, however, have not been used for evaluation of data from the gyroscope sensors. One of these methods is the method based on total length of trajectory in a 2-D plot, which is easily and intuitively understandable, and can also be used where the motion capture systems can only measure two sway angles, e.g. SwayStar system (Balance International Innovations $\mathrm{GmbH}$ ). These systems usually provide information on pitch and roll angles (Findling et al., 2011). However, this technique based on total length of trajectory in a 2-D plot of data recorded using the gyroscope sensors has never been used before to study postural stability.

The method based on total length of trajectory (i.e. length of center of pressure ( $\mathrm{CoP})$ path) in a 2-D plot is commonly accepted and verified in the field of quantitative evaluation of data obtained using a stabilometric platform (Donath, Roth, Zahner, \& Faude, 2012; Harbourne, Willett, Kyvelidou, Deffeyes, \& Stergiou, 2010; Raymakers et al., 2005). One of the main reasons behind this use of the method is that the study of the movement with one variable characterizing the change in two main angles may find new uses for the study of the postural stability by very inexpensive two axis gyroscopes which are also used in cell phones and electronic watches. It is generally known that the method based on evaluation of the data obtained using a gyroscope system could provide new information on balance deficits (Adkin et al., 2001; Allum et al., 2001;
Gill et al., 2001). The data processing based on the concept of the total length of the trajectory of pitch vs. roll angle might be a suitable tool. Thus, the aim of this work is to identify application possibilities of the length of the trajectory in a 2-D plot of the pitch angle vs. roll angle in clinical practice.

\section{Methods}

\section{Selection of participants}

In order to test the new method it is necessary to compare healthy participants without any postural balance problems to participants who have postural balance problems. A comparison of exclusively age groups would not be definite. The parameters of the body sway in healthy participants within the age span between 20 and 60 years vary only slightly, as studies have shown (Abrahámová \& Hlavačka, 2008; Aoki et al., 2012). It is generally known that the movement of the trunk (where the center of the mass of the whole body is located) and other body segments is directly related to the movement of the center of pressure which is traditionally used to study postural stability (Horak \& Hlavacka, 2001; Tia, Saimpon, Paizis, Mourey, Fadiga, \& Pozzo, 2011). Aoki et al. (2012) found that there were insignificant differences in 10-60 year old subjects in CoP sway parameters: circumference, rectangular area, left-right width, and front-back width in both the cases (eyes open/closed), i.e. no significant age-related differences were found in any of the CoP sway parameters (i.e. Romberg quotients). It has been demonstrated that a significant degradation of stability begins after the age of 60 . Also, a detailed analysis of an age-related increase of $\mathrm{CoP}$ parameters by the polynomial type of regression confirmed that a gradual increase of body sway characterized by an increase of CoP oscillations started after the age of 60 (Abrahámová \& Hlavačka, 2008).

Based on the above mentioned reasons, ten volunteer patients ( 6 men and 4 women; mean age of 52.2 (SD 11.7) years) with degenerative cerebellar ataxia and eleven healthy subjects ( 7 men and 4 women; mean age of 26.0 (SD 6.4) years) participated in the study. The degenerative ataxias are a heterogeneous group of hereditary and nonhereditary diseases described and studied in detail in Čakrt et al. (2012). They are characterized by progressive ataxia due to degeneration of the cerebellar cortex, cerebellar nuclei and spinal pathways, which results in impaired coordination of the limbs and postural instability. All Pts were recruited from the Department of Neurology at the Faculty Hospital Motol, Prague. A board-certified neurologist had previously screened and diagnosed cerebellar disorder. 
Diagnostic evaluation included a detailed disease history, a neurologic examination, routine laboratory blood and urine tests, and a magnetic resonance imaging of the brain. All Pts and HS expressed their informed consent before the experiment. HS were recruited from the students/volunteers at Charles University in Prague. In the case of the HS, the diagnostic evaluation included a detailed disease history and routine laboratory tests. The study was performed in accordance with the Helsinki Declaration. The study protocol was approved by the local ethical committee.

\section{Test procedure and measurement equipment}

The trunk and feet sway in the roll and pitch planes were measured while Pts and HS were standing with eyes open (EO) on a firm surface (FiS) and eyes closed (EC) on a soft foam surface (FoS). The reason for choosing these two most commonly used types of tests is to determine the difference between non-reduced visual perception and mechanoreceptor perception and maximum reduction of the visual perception and mechanoreceptor perception. These two tests also sufficiently demonstrate the applicability of the proposed technique of quantifying human postural stability and the differences between the behavior of the trunk and feet of healthy subjects and patients.

In brief, trunk (T) and feet (left foot (LF), right foot (RF)) sway were measured during stance. Subjects were standing upright barefoot on both feet on FiS for 60 seconds with eyes open (EO) and on FoS for 60 seconds with closed (EC). The participants' feet were positioned next to each other, splayed at $30^{\circ}$. Feet were spaced $5 \mathrm{~cm}$ apart, arms always in hanging position (Čakrt et al., 2012). A visual target was also positioned at eye level, approximately $1.5 \mathrm{~m}$ away from the subject. Measurement of each subject was performed after one minute of standing quietly on a specific type of surface that subject was accustomed to the type of surface before each trial.

For trunk and feet sway measurements, the system Xbus kit (Xsens, Enschede, Netherlands) was used. It is a lightweight device consists of datalogger (Xbus Master) and small 3DoF trackers (MTx units). It provides drift-free 3-D orientation. Therefore, the Xbus kit can also be seen as representative of gyroscope systems. The MTx unit was calibrated before each measurement, the MTx unit was set up in a way that the one axis of the coordinate system was parallel to the sagittal axis, i.e. symmetry axis of the platform on which the participants stood, and the other two axes were perpendicular to the sagittal axis with respect to the earth's gravity. The MTx unit was placed on patient's trunk in accordance with Ochi, Abe, Ishigami, Orsu, \& Tomita (1997), Adkin et al. (2001) and Warrenburg,
Bakker, Kremer, Bloem, and Allum (2005). The sensor was positioned at the level of the lower back (lumbar 2-3) near to the body's centre of mass (Adkin, Bloem, \& Allum, 2001; Warrenburg et al., 2005), see Figure 1. For the feet, the bases of the two MTx units are positioned and oriented over the dorsum of feet. In particular, it is recommended to place it over the midfoot (cuboid and cuneiforms) (Cutti, Ferrari, Garofalo, Raggi, Cappello, \& Ferrari, 2010). The MTx unit was firmly placed using double sided medical tape. The sway was measured with precision of $0.05^{\circ}$, the sample frequency was $100 \mathrm{~Hz}$. The MT Manager software (Xsens, Enschede, Netherlands) of the Xbus kit system was used to calculate the angles. The sensor fusion algorithm (Extended Kalman Filter) was mounted on the unit (Kalman, 1960). Sensors signal generation model is described directly by Xsens, see also (Luinge, 2002). The output signal was not filtered in any other way, to have the possibility of considering errors in final evaluation.

\section{Data analysis}

The new method to identify pathological balance control is based on the well-known mathematical tools used in static posturography (Raymakers et al., 2005). In our case, the total length (Stins, Ledebt, Emck, Dokkum, \& Beek, 2009; Teranishi et al., 2011) of trajectory (TL) is used to evaluate an angle-angle plot. The total length of pitch vs. roll is computed by calculating the sum of the Euclidean distances between consecutive data points (i.e. Cartesian coordinates) in Euclidean

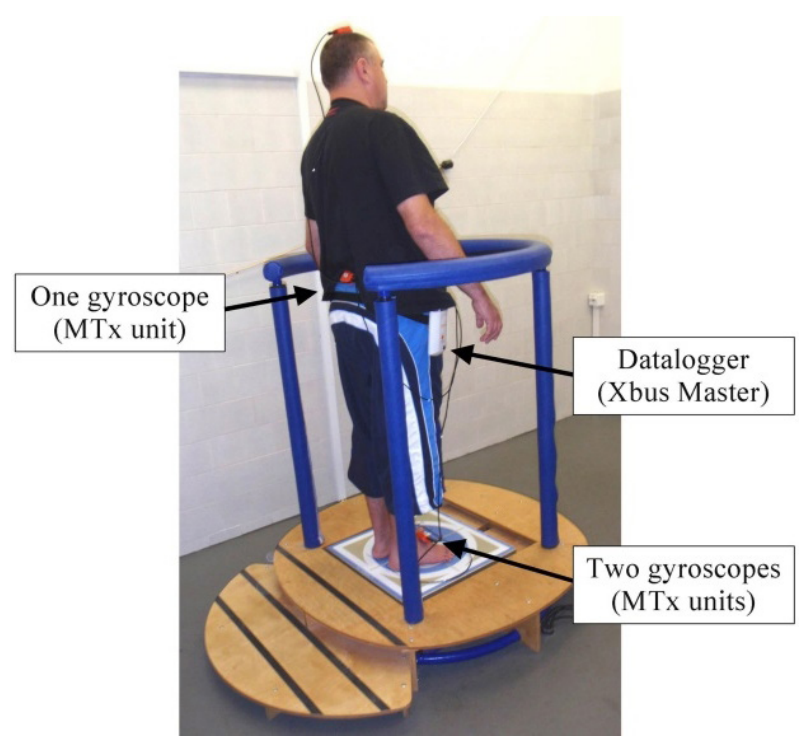

Figure 1. The gyroscope sensors (MTx units) used to measure the trunk and feet angular movements 
2-D space (Deza \& Deza, 2013) collected during the trial, given by Teranishi et al. (2011):

$T L=\sum_{n=1}^{N} \operatorname{dist}\left(P_{n}, P_{n-1}\right)=\sum_{n=1}^{N} \sqrt{\left(\alpha_{n}-\alpha_{n-1}\right)^{2}+\left(\beta_{n}-\beta_{n-1}\right)^{2}}$,

where the data point $P_{n}=\left(\alpha_{n^{\prime}} \beta_{n}\right)$ at time $n$ and the data point $P_{n-1}=\left(\alpha_{n-p} \beta_{n-1}\right)$ at time $n-1 . N$ is the number of sample points, $\alpha$ is the roll angle, $\beta$ is the pitch angle measured by a gyroscope sensor (Kim, Ferdjallah, \& Harris, 2009). The number of points is determined by the recorded length of the dataset (60 s) and the sample frequency $(100 \mathrm{~Hz})$. Thus, in our case, the TL is defined by the roll and pitch excursions when these variables are plotted as a 2-D plot, and the degree is the physical unit. Designed custom-written MATLAB program based on the functions of the MATLAB software (Version R2010b; Mathworks, Natick, MA, USA) was used to calculate the TLs, see Figure 2. The roll angle and pitch angle of each segment (trunk, left foot and right foot) were measured, and the TL of each segment was calculated using above mentioned method and software. TL of feet was calculated by making an average of TLs for each foot.

The reason for the use of the TLs is that the standardized parameters, such as the standard deviations of displacement, seem to be less transparent since they are not correlated to any of the trajectory parameters (Raymakers et al., 2005), which directly reflect the measured movement of a body segment (Vaz Garcia, 2009), and the TLs is widely used in the studies of the postural stability based on stabilometric platform (Raymakers et al., 2005).

\section{Statistical analysis}

After calculating the TLs of each subject standing on a FiS with $\mathrm{EO}$ and FoS with EC, the statistical analysis was performed using MATLAB software. The JarqueBera test (in MATLAB software) was used to test the normal distribution. The significance level was set at $5 \%$. The median, minimum, maximum, first quartile

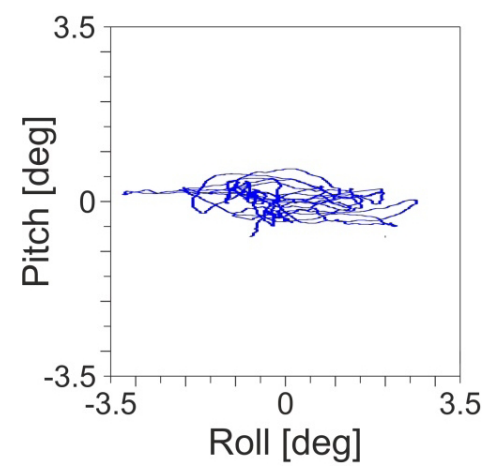

Figure 2. Example of a two-dimensional trajectory of the roll vs. pitch and third quartile of the TL are used to illustrate differences in groups of data. Also, the Wilcoxon rank sum test and Wilcoxon signed rank test were used to assess the significance of the differences between the results of these four groups: HS standing on a FiS with EO; HS standing on a FoS with EC; Pts standing on a FiS with EO; Pts standing on a FoS with EC. The significance level was set at $5 \%$. In addition, effect sizes, which consider the difference between two groups of data, were calculated in accordance with Fritz, Morris, and Richler (2012) and Coolican (2009).

\section{Results}

After calculating the TLs, the statistical analysis was performed, see Table 1. The following chart (Figure 3) compares the relationship between the TLs of the HS and Pts with EO and EC standing on a FiS or FoS. Since some data did not have a normal distribution, the Wilcoxon tests were used to analyze the data. Tests did not show significant difference in TLs of the left foot and right foot, the $p>.05$ in all cases. Therefore, in the further analysis, we assume no difference in movement of the left and right feet during standing.

In the case of TLs of the trunk of the HS with EO standing on a FiS and the Pts with EO standing on a FiS, the results show significant difference in TLs $(p=.02)$. In all other comparisons, the $p$ is less than .01 , thus the significant difference was found between TLs of the feet of the HS with EO standing on a FiS and the Pts with EO standing on a FiS, TLs of the trunk of the HS with EC standing on FoS and Pts with EC standing on FoS, TLs of the feet of the HS with EC standing on a FoS and the Pts with EC standing on a FoS.

In addition to testing the applicability of the method, we also examined whether the method identifies differences in postural stability under changing conditions. In the case of TLs of the trunk of the HS with EO standing on a FiS and the HS with EC standing on a FoS, the results show significant difference in TLs $(p=.02)$. In all other comparisons, the $p$ is less than .01 , thus the significant difference was found between TLs of the feet of the HS with EO standing on a FiS and the HS with EC standing on a FoS, TLs of the trunk of the Pts with EO standing on a FiS and the Pts with EC standing on a FoS, TLs of the feet of the Pts with EO standing on a FiS and the Pts with EC standing on a FoS. In all cases of comparison of the groups of data, the effect sizes (Cohen's $d$ ) were moderate to large, i.e. calculated values were greater than 0.5 (Cohen, 1988). 
Table 1

Descriptive statistics of the length of the trajectories of trunk, right foot and left foot of healthy subjects and patients standing on a firm surface with eyes open and foam surface with eyes closed

\begin{tabular}{|c|c|c|c|c|c|c|}
\hline & \multicolumn{3}{|c|}{ Healthy subjects } & \multicolumn{3}{|c|}{ Patients } \\
\hline & Trunk & Right foot & Left foot & Trunk & Right foot & Left foot \\
\hline \multicolumn{7}{|l|}{ Eyes open, firm surface } \\
\hline Minimum (deg) & 23 & 18 & 17 & 35 & 44 & 42 \\
\hline Maximum (deg) & 116 & 74 & 109 & 370 & 1272 & 769 \\
\hline Median (deg) & 39 & 35 & 39 & 98 & 115 & 105 \\
\hline First quartile (deg) & 27 & 30 & 25 & 70 & 102 & 88 \\
\hline Third quartile (deg) & 63 & 45 & 59 & 176 & 225 & 125 \\
\hline \multicolumn{7}{|l|}{ Eyes closed, foam surface } \\
\hline Minimum (deg) & 38 & 57 & 71 & 119 & 382 & 359 \\
\hline Maximum (deg) & 246 & 317 & 670 & 2859 & 2569 & 1228 \\
\hline Median (deg) & 73 & 100 & 165 & 576 & 1179 & 665 \\
\hline First quartile (deg) & 53 & 93 & 112 & 427 & 620 & 535 \\
\hline Third quartile (deg) & 139 & 200 & 238 & 712 & 1293 & 878 \\
\hline
\end{tabular}

A.

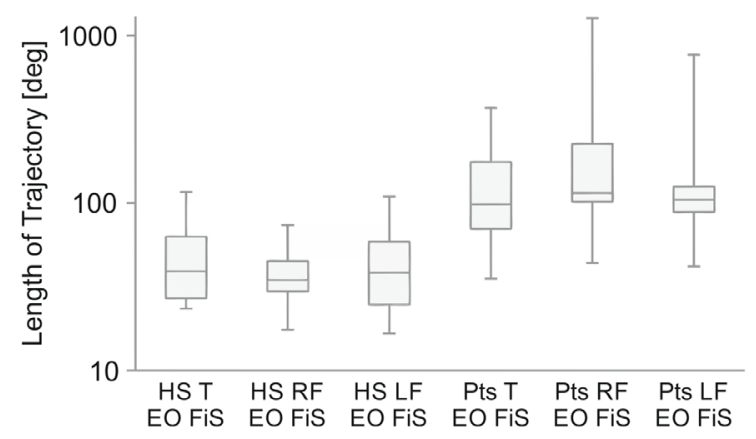

B.

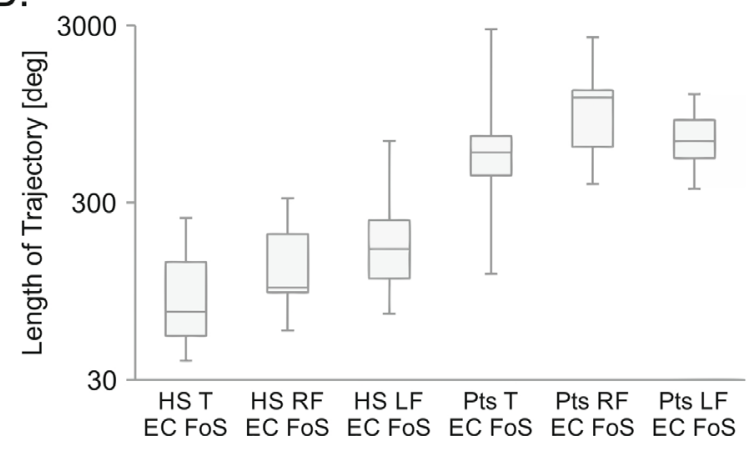

Figure 3. Comparison of the length of the trajectories of trunk (T), right foot (RF) and left foot (LF) of healthy subjects (HS) and patients (Pts) standing on a firm surface (FiS) with eyes open (EO) and foam surface $(\mathrm{FoS})$ with eyes closed (EC)

\section{Discussion}

The article aims to test the applicability of the method based on total length of trajectory in a 2-D plot of the pitch angle vs. roll angle. For this reason, we analyzed whether the method identifies differences in postural stability HS and Pts, and under changing conditions which are commonly used for studying the postural stability (Patel, Fransson, Lush, \& Gomez, 2008).

When the values of TLs for the Pts and HS were compared, statistically significant differences were found in all cases. It is apparent that in the case of the Pts, the increases in TLs are significant. Therefore, the findings are consistent with those obtained by traditional methods, which show significantly greater lengths of CoP path trajectories obtained using a stabilometric platform, or greater angular displacement and velocities obtained using a gyroscope, in the case of patients (Diener, Dichgans, Bacher, \& Gompf, 1984; Warrenburg et al., 2005). An interesting finding is that the greatest difference is in the case of measurement of TL of the trunk. The median of the TL of the trunk in Pts is 7.9 times larger than the median of the TL in HS with EC on a FoS (see Table 1). In case of measuring the feet, the differences in the calculated values of the medians are slightly smaller (see Table 1). The median of the TL of feet (left and right) in Pts is 5.6 times larger than the median of the TL in HS with EC on a FoS (see Table 1). Thus, results show that it is preferable to evaluate, in clinical practice, the measured data for the trunk than for the feet, because, in the case of the removing visual inputs and somatosensory inputs, the differences in postural stability are more pronounced.

In the case of the study of relationship between quiet stance trials with EO and EC, all calculated $p$-values were lower than the significance level. There is significant difference between the measured data of the 
participants with EO standing on FiS and participants with EC standing on FoS. This finding is also significantly evident from Figure 4. The measured values for the subjects with EC are significantly greater than in the case of the subjects with EO. In the case of the trunk, the median of the TL in HS with EC is only 1.9 times larger than the median of the TL in HS with EO, and the median of the TL in Pts with EC is 5.9 times larger than the median of the TL in Pts with EO (see Table 1). This finding justifies the reasons for the use of the standing with EC on a FoS in clinical practice, as is also mentioned in Blackburn, Riemann, Myers, and Lephart (2003).

If we look at the difference between HS and Pts in detail, the median of the TL of the trunk in Pts is 7.9 times larger than the median of the TL in HS with EC on a FoS. In the case of subjects with EO on a FiS, the median of the TL is only 2.5 times larger (see Table 1). Data analysis showed significant statistical differences between the two groups of subjects under each measurement condition. Some articles point to the possible effect of age on postural stability (Choy, Brauer, \& Nitz, 2003). In the mentioned study, certain instability was evident in women older than 50 years when a foam surface was introduced and in women older than 60 years when a firm surface was used. Similar conclusions are presented in Abrahámová and Hlavačka (2008), Aoki et al. (2012). Thus, it can be concluded that in the case of subjects standing on a FiS, the identified difference between HS and Pts is caused by the degenerative cerebellar disorder. The same cannot be applied with $100 \%$ certainty in the case of subjects standing on a FoS. In this case, the stability may be influenced partly by the age of the subject. However, it can be generally concluded that the new method is an appropriate tool to identify differences in balance control. Moreover, as it has been already mentioned, the results are in accordance with widely accepted results of the study of patients with cerebellar diseases obtained by a method based on total length of CoP path trajectory recorded using a stabilometric platform (Diener et al., 1984; Lucy \& Hayes, 1985).

There are limitations to our study. The most important one is that the sample of the subjects was small and probably not representative of the larger population. However, to test the basic attributes of the method proposed for the study of postural stability in this preliminary study, a sample of ten Pts and eleven HS is sufficient, just as it was in similar works (Rossi-Izquierdo et al., 2013). A second limitation in this study is that only one measurement of each subject was made. However, measurement of a subject with impaired postural stability is usually done only once, or only data from the trial with the most complete and longest record are used for further analysis (Honegger, Hillebrandt, Elzen, Tang, \& Allum, 2013; Horlings, Carpenter, Honegger, \& Allum, 2009). This approach was used because some patients had significant stability problems and were unable to perform multiple tests with EC, as is common in these patients (Čakrt et al., 2012).

\section{Conclusion}

It was found that the TL of the trajectory of pitch vs. roll in 2-D plot is suitable for quantifying postural sway and identifying differences in balance control. Although the results vary while measuring different segments of the body, the gyroscope system identified significant differences between two groups of subjects, middleaged patients with degenerative cerebellar disorder and
A.

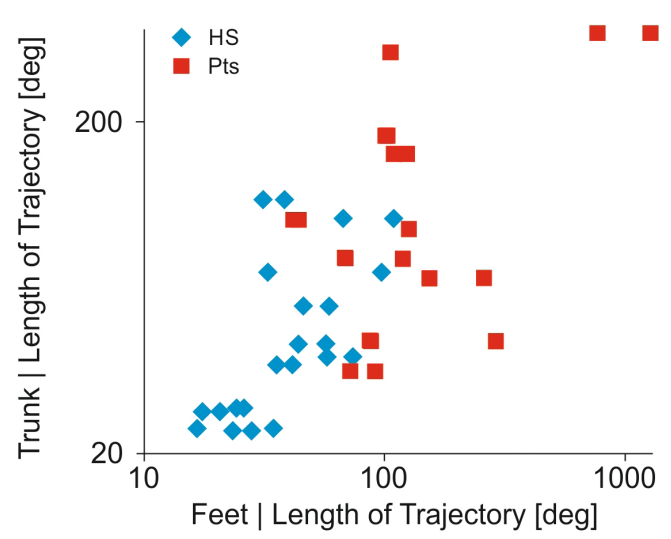

B.

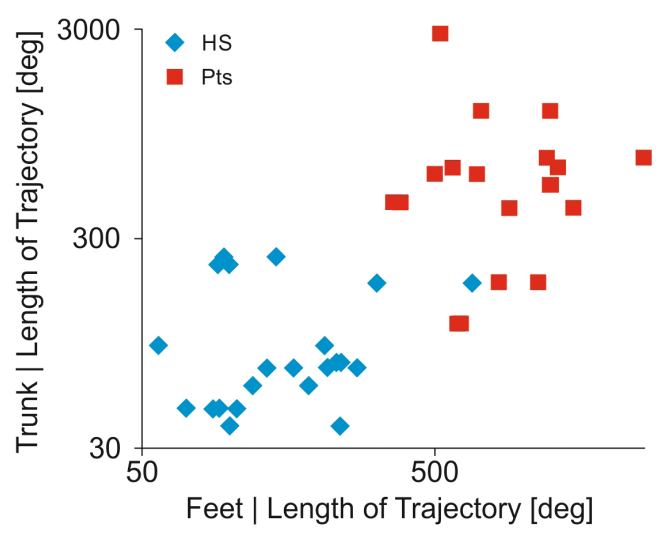

Figure 4. Comparison of the length of trajectory of the trunk and feet of HS and patients standing on a firm surface with eyes open (A.) and foam surface with eyes closed (B.) 
eleven young healthy subjects, in both cases of the measurement of the trunk and feet. Preliminary results also show that it is preferable to evaluate the measured data for the trunk than for feet, because the differences in the calculated values of the medians of TL between the two groups of subjects are greater. The major finding is that one cheap 2-DoF orientation tracker placed on patient's body segment, preferably on the trunk, in conjunction with the method based on the TL of pitch vs. roll could be a new tool for examinations of instability of the body segments during the stance tasks.

The concept of the TL, although familiar to the biomechanics community, has not been used before to study the postural instability using gyroscopic data. Further tests of the proposed method to assess the postural instability will be performed on more patients with different neurological disorders to compare and investigate reasons for similarities and differences in postural balance problems and abilities.

\section{Acknowledgment}

This work was done in the Joint Department of the Faculty of Biomedical Engineering CTU and Charles University in Prague in the framework of research program No.VG20102015002 (2010-2015, MV0/VG), sponsored by the Ministry of the Interior of the Czech Republic, and project SGS15/107/OHK4/1T/17 and SGS14/170/OHK4/2T/17 CTU Prague.

\section{References}

Abrahámová, D., \& Hlavačka, F. (2008). Age-related changes of human balance during quiet stance. Physiological Research, 57, 957-964.

Adkin, A. L., Bloem, B. R., \& Allum, J. H. J. (2001). Trunk sway measurements during stance and gait tasks in Parkinson's disease. Gait \& Posture, 22, 240-249.

Allum, J. H. J., Adkin, A. L., Carpenter, M. G., HeldZiolkowska, M., Honegger, F., \& Pierchala, K. (2001). Trunk sway measures of postural stability during clinical balance tests: Effects of a unilateral vestibular deficit. Gait \& Posture, 14, 227-237.

Aoki, H., Demura, S., Kawabata, H., Sugiura, H., Uchida, Y., Xu, N., \& Murase, H. (2012). Evaluating the effects of open/closed eyes and age-related differences on center of foot pressure sway during stepping at a set tempo. Advances in Aging Research, 1, 72-77.

Blackburn, J. T., Riemann, B. L., Myers, J. B., \& Lephart, S. M. (2003). Kinematic analysis of the hip and trunk during bilateral stance on firm, foam, and multiaxial support surfaces. Clinical Biomechanics, 18, 655-661.

Čakrt, O., Vyhnálek, M., Slabý, K., Funda, T., Vuillerme, N., Koláŕ, P., \& Jeřábek, J. (2012). Balance rehabilitation therapy by tongue electrotactile biofeedback in patients with degenerative cerebellar disease. NeuroRehabilitation, 31, 429-434.

Choy, N. L., Brauer, S., \& Nitz, J. (2003). Changes in postural stability in women aged 20 to 80 years. Journals of Gerontology Series A: Biological Sciences and Medical Sciences, 58, M525-M530.

Cohen, J. (1988). Statistical power analysis for the behavioral sciences. Hillsdale, NJ: Lawrence Erlbaum Associates.

Coolican, H. (2009). Research methods and statistics in psychology. London: Hodder \& Stoughton Publisher.

Cutti, A. G., Ferrari, A., Garofalo, P., Raggi, M., Cappello, A., \& Ferrari, A. (2010). 'Outwalk': A protocol for clinical gait analysis based on inertial and magnetic sensors. Medical \& Biological Engineering \& Computing, 48, 17-25.

Deza, M. M., \& Deza, E. (2013). Encyclopedia of distances. Berlin: Springer-Verlag.

Diener, H. C., Dichgans, J., Bacher, M., \& Gompf, B. (1984). Quantification of postural sway in normals and patients with cerebellar diseases. Electroencephalography and Clinical Neurophysiology, 57, 134-142.

Donath, L., Roth, R., Zahner, L., \& Faude, O. (2012). Testing single and double limb standing balance performance: Comparison of CoP path length evaluation between two devices. Gait \& Posture, 36, 439-443.

Duclos, C., Nadeau, S., \& Lecours, J. (2008). Lateral trunk displacement and stability during sit-to-stand transfer in relation to foot placement in patients with hemiparesis. Neurorehabilitation and Neural Repair, 6, 715-722.

Findling, O., Sellner, J., Meier, N., Allum, J. H. J., Vibert, D., Lienert, C., \& Mattle, H. P. (2011). Trunk sway in mildly disabled multiple sclerosis patients with and without balance impairment. Experimental Brain Research, 213, 363-370.

Fritz, C. O., Morris, P. E., \& Richler, J. J. (2012). Effect size estimates: Current use, calculations, and interpretation. Journal of Experimental Psychology: General, 141, 2-18.

Gill, J., Allum, J. H., Carpenter, M. G., Held-Ziolkowska, M., Adkin, A. L., Honegger, F., \& Pierchala, K. (2001). Trunk sway measures of postural stability during clinical balance tests: Effects of age. Journals of Gerontology Series A: Biological Sciences and Medical Sciences, 56, M438-M447.

Harbourne, R. T., Willett, S., Kyvelidou, A., Deffeyes, J., \& Stergiou, N. (2010). A comparison of interventions for children with cerebral palsy to improve sitting postural control: A clinical trial. Physical Therapy, 90, 1881-1898.

Honegger, F., Hillebrandt, I., Elzen, N., Tang, K., \& Allum, J. H. J. (2013). The effect of prosthetic feedback on the strategies and synergies used by vestibular loss subjects to control stance. Journal of NeuroEngineering and Rehabilitation, 115, 1-11.

Hong, S. L., Manor B., \& Li, L. (2007). Stance and sensory feedback influence on postural dynamics. Neuroscience Letters, 423, 104-108.

Horak, F. B., \& Hlavacka, F. (2001). Somatosensory loss increases vestibulospinal sensitivity. Journal of Neurophysiology, 86, 575-585.

Horlings, C. G. C., Carpenter, M. G., Honegger, F., \& Allum, J. H. J. (2009). Vestibular and proprioceptive contributions to human balance corrections: Aiding these with prosthetic feedback. Annals of the New York Academy of Sciences, 1164, 1-12. 
Horlings, C. G. C., Küng, U. M., Bloem, B. R., Honegger, F., Van Alfen, N., Van Engelen, B. G. M., \& Allum, J. H. J. (2008). Identifying deficits in balance control following vestibular or proprioceptive loss using posturographic analysis of stance tasks. Clinical Neurophysiology, 119, 2338-2346.

Hwang, I. S., Huang, C. T., Cherng, R. J., \& Huang, C. C. (2006). Postural fluctuations during pointing from a unilateral or bilateral stance. Human Movement Science, 25, 275-291.

Kalman, R. E. (1960). A new approach to linear filtering and prediction problems. Journal of Basic Engineering, 82, $35-45$.

Kammermeier, S., Kleine, J. F., Eggert, T., Krafczyk, S., \& Büttner, U. (2013). Disturbed vestibular-neck interaction in cerebellar disease. Journal of Neurology, 260, 794-804.

Kim, G., Ferdjallah, M., \& Harris, G. F. (2009). Fast computational analysis of sway area using center of pressure data in normal children and children with cerebral palsy. American Journal of Biomedical Sciences, 1, 364-372.

Lucy, S. D., \& Hayes, K. C. (1985). Postural sway profiles, normal subjects and subjects with cerebellar ataxia. Physiotherapy Canada, 37, 140-148.

Luinge, H. J. (2002). Inertial sensing of human movement. Enschede, Netherlands: Twente University Press.

Morris, M. E. (2000). Movement disorders in people with Parkinson disease: A model for physical therapy. Physical Therapy, 80, 578-597.

Ochi, F., Abe, K., Ishigami, S., Orsu, K., \& Tomita, H. (1997). Trunk motion analysis in walking using gyro sensors. In R. J. Jaeger (Ed.), Proceedings of the 19th Annual International Conference of the IEEE: Engineering in Medicine and Biology Society (pp. 1824-1825). Chicago, IL: IEEE.
Oliveira, L. F., Simpson, D. M., \& Nadal, J. (1996). Calculation of area of stabilometric signals using principal component analysis. Physiological Measurement, 17, 305-312.

Patel, M., Fransson, P. A., Lush, D., \& Gomez, S. (2008). The effect of foam surface properties on postural stability assessment while standing. Gait \& Posture, 28, 649-656.

Raymakers, J. A., Samson, M. M., \& Verhaar, H. J. J. (2005). The assessment of body sway and the choice of the stability parameter(s). Gait \& Posture, 21, 48-58.

Rossi-Izquierdo, M., Ernst, A., Soto-Varela, A., SantosPérez, S., Faraldo-García, A., Sesar-Ignacio, A., \& Basta, D. (2013). Vibrotactile neurofeedback balance training in patients with Parkinson's disease: Reducing the number of falls. Gait \& Posture, 37, 195-200.

Stins, J. F., Ledebt, A., Emck, C., Dokkum, E. H., \& Beek, P. J. (2009). Patterns of postural sway in high anxious children. Behavioral and Brain Functions, 5, 42.

Teranishi, T., Kondo, I., Sonoda, S., Wada, Y., Miyasaka, H., Tanino, G., ... Saitoh, E. (2011). Validity study of the standing test for imbalance and disequilibrium (SIDE): Is the amount of body sway in adopted postures consistent with item order? Gait \& Posture, 34, 295-299.

Tia, T., Saimpont, A., Paizis, C., Mourey, F., Fadiga, L., \& Pozzo, T. (2011). Does observation of postural imbalance induce a postural reaction? PLoS One, 6, Article ID e17799.

Vaz Garcia, F. (2009). Disequilibrium and its management in elderly patients. International Tinnitus Journal, 15, 83-90.

Warrenburg, B. P. C., Bakker, M., Kremer, B. P. H., Bloem, B. R., \& Allum, J. H. J. (2005). Trunk sway in patients with spinocerebellar ataxia. Movement Disorders, 20, 1006-1013. 